\title{
Is Cosmic Speed-Up Due to New Gravitational Physics?
}

\author{
Sean M. Carroll ${ }^{1 *}$, Vikram Duvvuri ${ }^{1 \dagger}$, Mark Trodden $^{2 \ddagger}$ and Michael S. Turner ${ }^{1,3,4 \S}$ \\ ${ }^{1}$ Enrico Fermi Institute, Department of Physics, \\ and Center for Cosmological Physics, University of Chicago, \\ 5640 S. Ellis Avenue, Chicago, IL 60637-1433, USA. \\ ${ }^{2}$ Department of Physics, Syracuse University, Syracuse, NY 13244-1130, USA \\ ${ }^{3}$ Department of Astronomy \& Astrophysics, University of Chicago, Chicago, IL 60637-1433, USA. \\ ${ }^{4}$ NASA/Fermilab Astrophysics Center, Fermi National Accelerator Laboratory, Batavia, IL 60510-0500, USA.
}

\begin{abstract}
We show that cosmic acceleration can arise due to very tiny corrections to the usual gravitational action of general relativity of the form $R^{n}$, with $n<0$. This eliminates the need for dark energy, though it does not address the cosmological constant problem. Since a modification to the EinsteinHilbert action of the form $R^{n}$, with $n>0$, can lead to early-time inflation, our proposal provides a unified and purely gravitational origin for the early and late time accelerating phases of the Universe.
\end{abstract}

Introduction. That the expansion of the Universe is currently undergoing a period of acceleration now seems inescapable: it is directly measured from the light-curves of several hundred type Ia supernovae 1, 2, 3], and independently inferred from observations of the cosmic microwave background (CMB) by the WMAP satellite 4] and other CMB experiments [5, 6 ].

Cosmic speed-up can be accommodated within general relativity by invoking a mysterious cosmic fluid with large negative pressure, dubbed dark energy. The simplest possibility for dark energy is a cosmological constant; unfortunately, the smallest estimates for its value are 55 orders of magnitude too large (for reviews see [7, 8]). This fact has motivated a host of other possibilities, most of which assume a zero cosmological constant, with the dynamical dark energy being associated with a new scalar field 9, 10, 11, 12, 13, 14, 15, 16, 17, 18, 19.

However, none of these suggestions is compelling and most have serious drawbacks. Given the challenge of this problem, it is worthwhile considering the possibility that cosmic acceleration is not due to some kind of stuff, but rather arises from new gravitational physics 20, 21, 22, 23, 24]. This is the angle we pursue in this letter.

General relativity is based upon the Einstein-Hilbert action, with Lagrange density $\sqrt{-g} R$, where $R$ is the curvature scalar. A natural modification is to add terms to the action that are proportional to $\sqrt{-g} R^{n}$. It is known that, for $n>1$, such terms lead to modifications of the standard cosmology at early times which lead to de Sitter behavior (Starobinskii inflation 25]). Here, we show that, for $n<0$, such corrections become important in the late Universe and can lead to self-accelerating vacuum solutions, providing a purely gravitational alternative to dark energy.

\footnotetext{
*carroll@theory.uchicago.edu

${ }^{\dagger}$ duvvuri@theory.uchicago.edu

¥trodden@physics.syr.edu

$\S$ mturner@oddjob.uchicago.edu
}

The self-accelerating solutions we find can behave like vacuum energy (i.e., $w_{\mathrm{DE}} \equiv P_{\mathrm{DE}} / \rho_{\mathrm{DE}}=-1$ ), or can lead to power-law acceleration with cosmic scale factor $a \propto t^{q}, q>1$, and $w_{\mathrm{DE}}<-2 / 3$. We also argue that our model is consistent with existing tests of gravitation theory and that the accelerating phase is consistent with current cosmological observations.

A Model. Many authors have considered modifying the Einstein-Hilbert action with terms that become effective in the high-curvature region. Here, however, we explore modifications which become important at extremely low curvatures to explain cosmic speed-up. For definiteness and simplicity we focus on the simplest correction to the Einstein-Hilbert action,

$$
S=\frac{M_{\mathrm{P}}^{2}}{2} \int d^{4} x \sqrt{-g}\left(R-\frac{\mu^{4}}{R}\right)+\int d^{4} x \sqrt{-g} \mathcal{L}_{M} .
$$

Here $\mu$ is a new parameter with units of [mass], $\mathcal{L}_{M}$ is the Lagrangian density for matter and the reduced Planck mass $M_{P} \equiv(8 \pi G)^{-1 / 2}$. For work on theories where the Lagrangian takes the form $f(R)$, see $26,27,28,29$, , 30 , 31, 32].

The field equation for the metric is then

$$
\begin{aligned}
(1+ & \left.\frac{\mu^{4}}{R^{2}}\right) R_{\mu \nu}-\frac{1}{2}\left(1-\frac{\mu^{4}}{R^{2}}\right) R g_{\mu \nu} \\
& +\mu^{4}\left[g_{\mu \nu} \nabla_{\alpha} \nabla^{\alpha}-\nabla_{(\mu} \nabla_{\nu)}\right] R^{-2}=\frac{T_{\mu \nu}^{M}}{M_{\mathrm{P}}^{2}},
\end{aligned}
$$

where $T_{\mu \nu}^{M}$ is the matter energy-momentum tensor.

The constant-curvature vacuum solutions, for which $\nabla_{\mu} R=0$, satisfy $R= \pm \sqrt{3} \mu^{2}$. Thus, we find the interesting result that the constant-curvature vacuum solutions are not Minkowski space, but rather are de Sitter space and anti de Sitter space. This bifurcation of the vacuum solutions from a unique one (Minkowski space) to two new ones (de Sitter and Anti-de Sitter spaces) as $\mu^{4}$ is increased from zero is unusual and is to be contrasted with the case of a cosmological constant. We will see that the de Sitter solution is, in fact, unstable, albeit with a very long decay time $\tau \sim \mu^{-1}$. 
We consider a perfect-fluid energy-momentum tensor,

$$
T_{\mu \nu}^{M}=\left(\rho_{M}+P_{M}\right) U_{\mu} U_{\nu}+P_{M} g_{\mu \nu},
$$

where $U^{a}$ is the fluid rest-frame four-velocity, $\rho_{M}$ is the energy density, $P_{M}$ is the pressure and we write $P_{M}=w \rho_{M}$. Matter corresponds to $w=0$ and radiation to $w=1 / 3$. We take the metric to be of the flat Robertson-Walker form, $d s^{2}=-d t^{2}+a^{2}(t) d \mathbf{x}^{2}$, for which the curvature scalar satisfies

$$
R=6\left[\frac{\ddot{a}}{a}+\left(\frac{\dot{a}}{a}\right)^{2}\right]=6\left(\dot{H}+2 H^{2}\right),
$$

where an overdot denotes differentiation with respect to time, $H=\dot{a} / a$ and $a(t)$ is the scale factor.

The time-time component of the field equations for this metric is

$$
\begin{aligned}
3 H^{2} & -\frac{\mu^{4}}{12\left(\dot{H}+2 H^{2}\right)^{3}}(2 H \ddot{H} \\
& \left.+15 H^{2} \dot{H}+2 \dot{H}^{2}+6 H^{4}\right)=\frac{\rho_{M}}{M_{\mathrm{P}}^{2}} .
\end{aligned}
$$

This replaces the usual Friedmann equation, recovered by setting $\mu=0$. The space-space components of (2) lead to the other independent Einstein equation,

$$
\begin{gathered}
\dot{H}+\frac{3}{2} H^{2}-\frac{\mu^{4}}{72\left(\dot{H}+2 H^{2}\right)^{2}}\left[4 \dot{H}+9 H^{2}\right. \\
\left.-R^{2} \partial_{0} \partial_{0}\left(\frac{1}{R^{2}}\right)-2 R^{2} H \partial_{0}\left(\frac{1}{R^{2}}\right)\right]=-\frac{P_{M}}{2 M_{\mathrm{P}}^{2}}
\end{gathered}
$$

The above fourth-order equations are complicated and it is difficult to extract details about cosmological evolution from them. It is convenient to transform from our current frame, which we call the matter frame, to the Einstein frame, where the gravitational Lagrangian takes the Einstein-Hilbert form and the additional degrees of freedom $(\ddot{H}$ and $\dot{H})$ are represented by a fictitious scalar field $\phi$.

Following 29], we make a conformal transformation

$$
\tilde{g}_{\mu \nu}=p(\phi) g_{\mu \nu}, \quad p \equiv \exp \left(\sqrt{\frac{2}{3}} \frac{\phi}{M_{\mathrm{P}}}\right) \equiv 1+\frac{\mu^{4}}{R^{2}},
$$

where $\phi$ is a real scalar function on space-time, $d \tilde{t}=$ $\sqrt{p} d t$, and $\tilde{a}(t)=\sqrt{p} a(t)$. It is then convenient to define an Einstein-frame matter energy-momentum tensor by

$$
\tilde{T}_{\mu \nu}^{M}=\left(\tilde{\rho}_{M}+\tilde{P}_{M}\right) \tilde{U}_{\mu} \tilde{U}_{\nu}+\tilde{P}_{M} \tilde{g}_{\mu \nu},
$$

where $\tilde{U}_{a} \equiv \sqrt{p} U_{a}, \tilde{\rho}_{M}=\rho_{M} / p^{2}$ and $\tilde{P}_{M}=P_{M} / p^{2}$.

In terms of the new metric $\tilde{g}_{\mu \nu}$, our theory is that of a scalar field $\phi\left(x^{\mu}\right)$ minimally coupled to Einstein gravity, and non-minimally coupled to matter, with potential

$$
V(\phi)=\mu^{2} M_{\mathrm{P}}^{2} \frac{\sqrt{p-1}}{p^{2}},
$$

shown in the figure below. Note that $V(\phi) \rightarrow 0$ both for $\phi \rightarrow 0(p \rightarrow 1)$ and for $\phi \rightarrow \infty(p \rightarrow \infty)$ and achieves its maximum, $9 \mu^{2} M_{\mathrm{P}}^{2} / 16 \sqrt{3}$, at $p=4 / 3$.

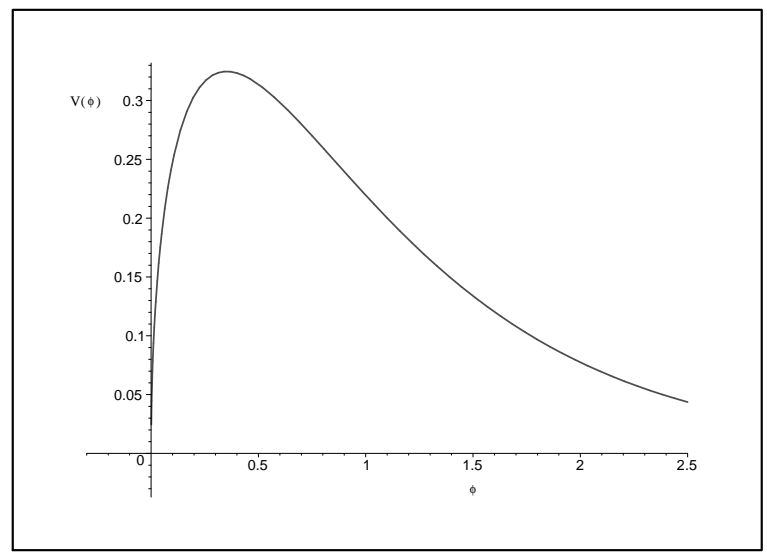

FIG. 1: The Einstein-frame potential $V(\phi)$

Denoting with a tilde all quantities (except $\phi$ ) in the Einstein frame, the relevant Einstein-frame cosmological equations of motion are

$$
\begin{gathered}
3 \tilde{H}^{2}=\frac{1}{M_{\mathrm{P}}^{2}}\left[\rho_{\phi}+\tilde{\rho}\right], \\
\phi^{\prime \prime}+3 \tilde{H} \phi^{\prime}+\frac{d V}{d \phi}(\phi)-\frac{(1-3 w)}{\sqrt{6}} \tilde{\rho}_{M}=0,
\end{gathered}
$$

where a prime denotes $d / d \tilde{t}$, and where

$$
\tilde{\rho}_{M}=\frac{C}{\tilde{a}^{3(1+w)}} \exp \left[-\frac{(1-3 w)}{\sqrt{6}} \frac{\phi}{M_{\mathrm{P}}}\right],
$$

with $C$ a constant, and

$$
\rho_{\phi}=\frac{1}{2} \phi^{\prime 2}+V(\phi) .
$$

The matter-frame Hubble parameter $H$ is related to that in the Einstein frame $\tilde{H} \equiv \tilde{a}^{\prime} / \tilde{a}$ by

$$
H=\sqrt{p}\left(\tilde{H}-\frac{\phi^{\prime}}{M_{\mathrm{P}} \sqrt{6}}\right) .
$$

What can we say about cosmological solutions in the more friendly setting of the Einstein frame? Ordinarily, Einstein gravity with a scalar field with a minimum at $V=0$ would yield a Minkowski vacuum state. However, here this is no longer true. Even though $V \rightarrow 0$ as $\phi \rightarrow$ 0 , this corresponds to a curvature singularity and so is not a Minkowski vacuum. The other minimum of the potential, at $\phi \rightarrow \infty$, is clearly not a static solution. These statements are also easily seen in the matter frame.

Now let us first focus on vacuum cosmological solutions, i.e., $P_{M}=\rho_{M}=0$. The beginning of the Universe corresponds to $R \rightarrow \infty$ and $\phi \rightarrow 0$. The initial conditions 
we must specify are the initial values of $\phi$ and $\phi^{\prime}$, denoted as $\phi_{i}$ and $\phi_{i}^{\prime}$. For simplicity we take $\phi_{i} \ll M_{\mathrm{P}}$. There are then three qualitatively distinct outcomes, depending on the value of $\phi_{i}^{\prime}$.

1. Eternal de Sitter. There is a critical value of $\phi_{i}^{\prime} \equiv \phi_{C}^{\prime}$ for which $\phi$ just reaches the maximum of the potential $V(\phi)$ and comes to rest. In this case the Universe asymptotically evolves to a de Sitter solution (ignoring spatial perturbations). As we have discovered before (and is obvious in the Einstein frame), this solution requires tuning and is unstable, since any perturbation will induce the field to roll away from the maximum of its potential.

2. Power-Law Acceleration. For $\phi_{i}^{\prime}>\phi_{C}^{\prime}$, the field overshoots the maximum of $V(\phi)$. Soon thereafter, the potential is well-approximated by $V(\phi) \simeq$ $\mu^{2} M_{\mathrm{P}}^{2} \exp \left(-\sqrt{3 / 2} \phi / M_{\mathrm{P}}\right)$, and we easily solve for $\tilde{a}(\tilde{t}) \propto$ $\tilde{t}^{4 / 3}$, which corresponds to $a(t) \propto t^{2}$ in the matter frame. Thus, the Universe evolves to late-time power-law inflation, with observational consequences similar to dark energy with equation-of-state parameter $w_{\mathrm{DE}}=-2 / 3$.

3. Future Singularity. For $\phi_{i}^{\prime}<\phi_{C}^{\prime}$, $\phi$ does not reach the maximum of its potential and rolls back down to $\phi=$ 0 . This yields a future curvature singularity in which the Hubble parameter remains finite in both the matter and Einstein frames, but its time derivative becomes infinite because $\dot{H} \propto V^{\prime} \rightarrow 1 / \sqrt{\phi} \rightarrow \infty$.

We now turn to the more interesting case in which the Universe contains matter. As can be seen from (10), the major difference here is that the equation-of-motion for $\phi$ in the Einstein frame has a new term. Furthermore, since the matter density is much greater than $V \sim \mu^{2} M_{\mathrm{P}}^{2}$ for $t \ll 14 \mathrm{Gyr}$, this term is very large and greatly affects the evolution of $\phi$. The exception is when the matter content is radiation alone $(w=1 / 3)$, in which case it decouples from the $\phi$ equation due to conformal invariance.

Despite this complication, it is possible to show that the three possible cosmic futures identified in the vacuum case remain in the presence of matter. To see this, first note that, if we ignore the $V^{\prime}$ term in (10) because it is subdominant at early times, and assume that $3 \tilde{H}^{2}=$ $\tilde{\rho}_{M} / M_{\mathrm{P}}^{2}$, we obtain

$$
\phi^{\prime}(\tilde{t})=\phi^{\prime}\left(\tilde{t}_{0}\right)\left(\frac{\tilde{a}_{0}}{\tilde{a}}\right)^{3}+\frac{C}{\sqrt{6}}\left(\frac{\tilde{t}-\tilde{t}_{0}}{\tilde{a}^{3}}\right)
$$

for $w=0$, and

$$
\phi^{\prime}(\tilde{t})=\phi^{\prime}\left(\tilde{t}_{0}\right)\left(\frac{\tilde{a}_{0}}{\tilde{a}}\right)^{3},
$$

for $w=1 / 3$.

The value of $\phi^{\prime}$ redshifts as it would for a free scalar field and, for $w=0$, increases dramatically due to the presence of matter. By tuning $\phi_{i}^{\prime}$, the value of $\phi^{\prime}$ at the epoch when matter becomes unimportant (i.e.,
$\tilde{\rho}_{M}<\mu^{2} M_{\mathrm{P}}^{2}$ ) can be adjusted, resulting in the same three futures as in the vacuum theory.

Thus far, we have left the dimensionful parameter $\mu$ unspecified beyond the requirement that it be sufficiently small that precision tests of gravity theory are not affected (see below). By choosing $\mu \sim 10^{-33} \mathrm{eV}$, the corrections to the standard cosmology only become important at the present epoch, making our theory a candidate to explain the observed acceleration of the Universe without recourse to dark energy. It is, of course, important to be clear that we have merely chosen the value of $\mu$ to achieve this end. Since we have no particular reason for this choice, such a tuning appears no more attractive than the traditional choice of the cosmological constant. However, it is intriguing to note that, in the present circumstance, the smallness of the $1 / R$ term in the action is directly related to the lateness of the accelerating phase. Thus, even an extremely tiny correction to the Einstein action can eventually have dramatic consequences.

Clearly our choice of correction to the gravitational action can be generalized. Terms of the form $-\mu^{2(n+1)} / R^{n}$, with $n>1$, lead to similar late-time self acceleration. Such actions can be transformed into the Einstein frame with scalar field potential

$$
V(p)=\mu^{2} M_{\mathrm{P}}^{2}\left(\frac{n+1}{2 n}\right) n^{1 /(n+1)} \frac{(p-1)^{n /(n+1)}}{p^{2}},
$$

with resulting matter-frame scale factor $a(t) \propto t^{q}$, where

$$
q=\frac{(2 n+1)(n+1)}{n+2} .
$$

Such a modification thus yields behavior similar to a dark energy component with equation of state parameter

$$
w_{\text {eff }}=-1+\frac{2(n+2)}{3(2 n+1)(n+1)} .
$$

As $n \rightarrow \infty$ the expansion approaches an exponential and the space-time is approximately de Sitter. Clearly therefore, such modifications can easily accommodate current observational bounds [33, 34] on the equation of state parameter $-1.45<w_{\mathrm{DE}}<-0.74(95 \%$ confidence level).

Finally, any modification of the Einstein-Hilbert action must, of course, be consistent with the classic solar system tests of gravity theory, as well as numerous other astrophysical dynamical tests. Many such tests depend on the Schwarzschild solution, which Birkhoff's theorem ensures is the unique, static, spherically-symmetric solution. It is simple to prove a related result here: that the most general, static, spherically-symmetric solutions are the Schwarzschild-(anti) de Sitter solutions. These solutions differ, of course, from the familiar Schwarzschild solution, which asymptotically approaches Minkowski space. However, it is clear that, if the parameter $\mu^{4}$ is chosen small enough, any astrophysical tests of gravity 
that depend on the Schwarzschild solution will be unaffected by the modification we have made. We expect similar arguments will hold for the cases of charged black holes and those with angular momentum.

Concluding Remarks. We have shown that the current epoch of cosmic acceleration can arise through purely gravitational effects, eliminating the need for dark energy. In particular, modifications to the Einstein-Hilbert action of the form $-\mu^{2(n+1)} / R^{n}$, with $\mu \sim 10^{-33} \mathrm{eV}$ and $n<0$, can lead to cosmic speed up with $-1<w_{\text {eff }} \leq$ $-2 / 3$.

There is, of course, growing evidence from measurements of CMB anisotropy, as well as of large-scale structure, that the Universe also evolved through a period of accelerated expansion very early on, known as inflation. It is also known that modifications to the EinsteinHilbert action of the form $m^{-2} R^{2}$, with $m$ a mass parameter, yield an Einstein-frame potential

$$
V(\phi)=m^{2} M_{\mathrm{P}}^{2} \frac{(p-1)^{2}}{8 p},
$$

where, as before, $p \equiv \exp \left[\sqrt{2 / 3} \phi / M_{\mathrm{P}}\right]$. In this case, the beginning of the Universe occurs at large $\phi$ and the Universe undergoes inflation. Thus, our work implies that it is possible for both eras of cosmic acceleration to arise from the gravitational sector of the theory, with general relativity being a valid description of the Universe only at intermediate cosmic times.

There are certainly many other details of this theory that remain to be checked. In particular, we would like to analyze quantitatively the behavior of the transition from matter domination to self acceleration as well as the growth of linearized perturbations about the given background solutions we have identified.

We thank C. Armendariz-Picon, T. Beloreshka, M. Bowick and Laura Mersini for extensive discussions and R. Brandenberger, E. Lim, D. Marolf, R. Myers, T. Jacobson and T. Vachaspati for helpful comments. The work of SMC is supported in part by the DOE, the NSF, and the Packard Foundation. VD is supported in part by the NSF. MT is supported in part by the NSF under grant PHY-0094122, and is a Cottrell Scholar of Research Corporation. MST is supported in part by the US DOE (at Chicago), the NASA (at Fermilab), and the NSF (at Chicago).

[1] A. G. Riess et al. [Supernova Search Team Collaboration], Astron. J. 116, 1009 (1998) arXiv:astro-ph/9805201.

[2] S. Perlmutter et al. [Supernova Cosmology Project Collaboration], Astrophys. J. 517, $565 \quad$ (1999) arXiv:astro-ph/9812133.
[3] J. L. Tonry et al., arXiv:astro-ph/0305008

[4] C. L. Bennett et al., arXiv:astro-ph/0302207

[5] C. B. Netterfield et al. [Boomerang Collaboration], Astrophys. J. 571, 604 (2002) arXiv:astro-ph/0104460.

[6] N. W. Halverson et al., Astrophys. J. 568, 38 (2002) arXiv:astro-ph/0104489.

[7] S. M. Carroll, Living Rev. Rel. 4, 1 (2001) arXiv:astro-ph/0004075.

[8] P. J. Peebles and B. Ratra, Rev. Mod. Phys. 75, 599 (2003) arXiv:astro-ph/0207347.

[9] C. Wetterich, Nucl. Phys. B 302, 668 (1988).

[10] B. Ratra and P. J. Peebles, Phys. Rev. D 37, 3406 (1988).

[11] R. R. Caldwell, R. Dave and P. J. Steinhardt, Phys. Rev. Lett. 80, 1582 (1998) arXiv:astro-ph/9708069.

[12] C. Armendariz-Picon, T. Damour and V. Mukhanov, Phys. Lett. B 458, 209 (1999) [arXiv:hep- th/9904075].

[13] C. Armendariz-Picon, V. Mukhanov and P. J. Steinhardt, Phys. Rev. Lett. 85, $4438 \quad$ (2000) arXiv:astro-ph/0004134.

[14] C. Armendariz-Picon, V. Mukhanov and P. J. Steinhardt, Phys. Rev. D 63, 103510 (2001) arXiv:astro-ph/0006373.

[15] L. Mersini, M. Bastero-Gil and P. Kanti, Phys. Rev. D 64, 043508 (2001) arXiv:hep-ph/0101210.

[16] R. R. Caldwell, Phys. Lett. B 545, 23 (2002) arXiv:astro-ph/9908168.

[17] S. M. Carroll, M. Hoffman and M. Trodden, arXiv:astro-ph/0301273

[18] V. Sahni and A. A. Starobinsky, Int. J. Mod. Phys. D 9, 373 (2000) arXiv:astro-ph/9904398.

[19] L. Parker and A. Raval, Phys. Rev. D 60, 063512 (1999) arXiv:gr-qc/9905031.

[20] C. Deffayet, G. R. Dvali and G. Gabadadze, Phys. Rev. D 65, 044023 (2002) arXiv:astro-ph/0105068.

[21] K. Freese and M. Lewis, Phys. Lett. B 540, 1 (2002) arXiv:astro-ph/0201229.

[22] M. Ahmed, S. Dodelson, P. B. Greene and R. Sorkin, arXiv:astro-ph/0209274

[23] N. Arkani-Hamed, S. Dimopoulos, G. Dvali and G. Gabadadze, arXiv:hep-th/0209227

[24] G. Dvali and M. S. Turner, arXiv:astro-ph/0301510

[25] A. A. Starobinsky, Phys. Lett. B 91, 99 (1980).

[26] J. D. Barrow and A. C. Ottewill, J. Phys. A 16, 2757 (1983).

[27] J. D. Barrow and S. Cotsakis, Phys. Lett. B 214, 515 (1988).

[28] J. D. Barrow and S. Cotsakis, Phys. Lett. B 258, 299 (1991).

[29] G. Magnano and L. M. Sokolowski, Phys. Rev. D 50, 5039 (1994) arXiv:gr-qc/9312008.

[30] A.Dobado and A.L. Maroto, Phys. Rev. D 52, 1895 (1995).

[31] H. J. Schmidt, Astron. Nachr. 311, 165 (1990) arXiv:gr-qc/0109004.

[32] S. Capozziello, S. Carloni and A. Troisi, arXiv:astro-ph/0303041

[33] A. Melchiorri, L. Mersini, C. J. Odman and M. Trodden, arXiv:astro-ph/0211522

[34] D. N. Spergel et al., arXiv:astro-ph/0302209 